\title{
Cell cycle regulator geminin is dispensable for the proliferation of vascular smooth muscle cells
}

\author{
GUO JianQiang \& SUN NingLing* \\ Department of Cardiology, Peking University People's Hospital, Beijing 100044, China
}

Received January 28, 2013; accepted June 4, 2013; published online July 8, 2013

\begin{abstract}
The proliferation of vascular smooth muscle cells (VSMCs) plays a major role in the pathogenesis of many cardiovascular diseases. Geminin regulates DNA replication and cell cycle progression and plays a key role in the proliferation of cancer cells. We therefore hypothesized that geminin regulates the proliferation of VSMCs. The present study demonstrates that the level of geminin expression was low in quiescent VSMCs (approximately $90 \%$ and $10 \%$ of cells in the G1 and in S/G2/M phases of the cell cycle, respectively), increased as more cells entered in S/G2/M, and then decreased as cells exited S/G2/M. Further, angiotensin II and norepinephrine stimulated expression of geminin in VSMCs. However, the DNA content, nuclear morphology, percentage of cells at different stages of the cell cycle, and rate of proliferation of VSMCs from which geminin was either depleted or overexpressed were all similar. These findings indicate geminin functions differently in VSMCs than it does in cancer cell lines and that it may provide a target for treating cancers without affecting normal cells.
\end{abstract}

geminin, DNA replication, cell cycle, proliferation, vascular smooth muscle cell

Citation: Guo J Q, Sun N L. Cell cycle regulator geminin is dispensable for the proliferation of vascular smooth muscle cells. Sci China Life Sci, 2013, 56: 731-738, doi: 10.1007/s11427-013-4513-1

The proliferation of vascular smooth muscle cell (VSMCs) is adaptive and essential for vasculogenesis and vascular repair after injury. However, excessive proliferation contributes to the pathogenesis of many cardiovascular diseases, including atherosclerosis, restenosis after angioplasty, and hypertension [1,2]. Although a large number of molecules, including inflammatory cytokines and growth factors, can promote the proliferation of VSMCs, therapeutic strategies targeting cell cycle may provide the best approach for inhibiting proliferation [3], because all proliferating cells must transit the cell cycle.

A critical function of the cell cycle is to ensure that the chromosomal DNA is replicated faithfully and completely and is followed by mitosis once in each cell cycle. When DNA replication initiates in eukaryotes, the origin recogni-

*Corresponding author (email: nlsun@263.net) tion complex binds to initiator elements and recruits the cell division cycle 6 and chromatin licensing and DNA replication factor 1 (Cdt1) to chromatin in the G1 phase of the cell cycle. This is followed by the loading of a replicative helicase (minichromosome maintenance complex components, MCM) onto chromatin that binds to cell division cycle 6 and Cdt1 to form the pre-replication complex (pre-RC), which unwinds chromosomes at replication forks and initiates DNA synthesis. After DNA replication origin firing at the beginning of $\mathrm{S}$ phase, the pre-RC dissociates from chromatin and is not reassembled until the cell passes through mitosis into the G1 phase of the next cycle [4-6].

Geminin, a cell cycle regulator, prevents DNA rereplication by preventing the loading of the MCM complex on chromatin to safeguard genomic stability [7]. Geminin is degraded during G1, accumulates during S, G2, and M, and is degraded at the metaphase-anaphase transition [8]. Sub- 
sequently, a study found that geminin blocks the binding of the MCM complex to replication origins primarily by binding with high affinity to Cdt1 during S and G2 [6]. Depletion of geminin by using RNA interference induces DNA rereplication as manifested by the appearance of giant nuclei and increased DNA content (greater than $4 \mathrm{~N}$ ). DNA rereplication induced by depletion of geminin activates the Chk1 and Chk2 G2/M checkpoints, which prevents cells with over-replicated DNA from entering mitosis and subsequently inhibits cell proliferation $[7,9,10]$.

Overexpression of geminin in a human breast non-tumorigenic cell line and in breast cancer cell line stimulates progression through G-S as well as cell proliferation [11]. Further, geminin is expressed at low levels in organs with minimal proliferative activity capacity (heart, nerves, prostate, kidney, lung, and skeletal muscle) and at high levels in proliferating lymphocytes, male germ cells, and epithelial cells [12-14]. This suggests that geminin acts as a regulator of cell proliferation. We therefore hypothesize that geminin regulates the proliferation of VSMCs by regulating DNA replication and cell cycle progression.

\section{Materials and methods}

\subsection{Construction of geminin protein and shRNA ex- pression vectors}

To isolate a geminin cDNA from rat cells, forward (5'-CGGAATTCATGAATCTCAGTATGAAGCAGAAACAGG-3') and reverse (5'-CGGGATCCCCTGTACACGCCGTGCCATC- $3^{\prime}$ ) primers were designed (accession No. NM_001106112.1). A standard reverse transcriptase polymerase chain reaction (PCR) was performed using RNA isolated from the rat A10 aortic smooth muscle cells line (CRL 1476, American Type Culture Collection, Manassas, VA, USA) as template. The purified products were ligated to a mammalian vector that expresses enhanced green fluorescent protein $\mathrm{pEGFP}-\mathrm{N} 1$ to generate a recombinant vector designated pEGFP-N1-Geminin. Three siRNAs targeting geminin and a negative control siRNA were synthesized at Shanghai GenePharma Co., Ltd. (Shanghai, China). The double-stranded siRNA sequences are as follows: Geminin-rat-738: 5'-GCCUGAGAAAGGAGAAUAATT-3', 5'UUAUUCUCCUUUCUCAGGCTT-3'; Geminin-rat-417: 5'-GGAGAACGCUGAAGAUGAUTT-3', 5'-AUCAUCUUCAGCGUUCUCCTT-3'; Geminin-rat-658: 5'-GAGGAAAGCUCUCUAUGAATT-3', 5'-UUCAUAGAGAGCUUUCCUCTT-3'.

We cotransfected 293T cells with pEGFP-N1-Geminin together with each siRNA and Lipofectamine 2000 (Invitrogen, USA) and selected the most inhibitory siRNA according to observations using a fluorescence microscope. A single-stranded hairpin siRNA (shRNA) vector based on the most effective siRNA was constructed at the SinoGenoMax
Co., Ltd. (Beijing, China). An adenovirus expressing this shRNA and the gene encoding geminin were also constructed at the SinoGenoMax Co., Ltd.

\subsection{Cell culture}

The A-10 rat aortic smooth muscle cells line and HeLa cells (CCL-2, American Type Culture Collection, Manassas, VA, USA) were grown in Dulbecco's modified Eagle's medium (DMEM, Life Technologies, Carlsbad, CA, USA) supplemented with $10 \%$ fetal bovine serum (FBS), $100 \mathrm{U} \mathrm{mL}^{-1}$

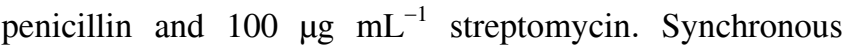
populations of quiescent VSMCs were obtained by incubating cells with DMEM containing 0.5\% FBS for $24 \mathrm{~h}$. Cells were then treated with DMEM containing 10\% FBS and harvested at $0,9,12,15,18,24 \mathrm{~h}$ following serum restimulation to determine geminin expression. Synchronized VSMCs cultured in DMEM containing 0.5\% FBS were also treated with angiotensin II (Ang-II), norepinephrine (NE) and were harvested at $24 \mathrm{~h}$. Ang-II and NE were purchased from Sigma Chemical Co. (St. Louis, MO, USA).

VSMCs were infected with adenoviral vectors (Ad-null, Ad-geminin Ad-non-silence, Ad-shRNA) at a multiplicity of infection of 200. The medium was then changed to DMEM containing $10 \%$ FBS and the cells were harvested at 0,24 and $48 \mathrm{~h}$ for the analyses as described in the following sections.

\subsection{Western blot analysis}

Western blot analysis of whole cell lysates was performed as previously described [15]. Briefly, the cells were lysed in RIPA buffer (P0013C, Beyotime Institute of Biotechnology, China). The cell lysates were centrifuged at $13000 \times g$ at $4^{\circ} \mathrm{C}$ for $20 \mathrm{~min}$ and the supernatant was collected. The total protein concentrations were measured with a BCA protein assay kit (Pierce, USA) according to the manufacturer's instructions. Electrophoresis of equal amounts of total protein was on a 10\% SDS-PAGE gel. Transfer was done in wet conditions. The primary antibodies were incubated overnight at $4^{\circ} \mathrm{C}$. Horseradish peroxidase (HRP)-conjugated secondary antibody was incubated for $2 \mathrm{~h}$. Antibodies purchased from Santa Cruz Biotechnology, Inc. (Santa Cruz, CA, USA) are as follows (dilutions, catalog numbers): geminin $(1: 500)$ (sc-74496), GAPDH (1:500) (sc-137179) and HRP-conjugated secondary antibody $(1: 3000)$ (sc2005). The GAPDH antibody served as internal control.

\subsection{Flow cytometry and cell proliferation assays}

Cells were collected by trypsinization, and washed with phosphate-buffered saline (PBS), fixed with $70 \%$ ethanol overnight at $4^{\circ} \mathrm{C}$. After fixation, cells were collected by centrifugation and incubated with RNaseA at $37^{\circ} \mathrm{C}$ for $30 \mathrm{~min}$, then stained with propidium iodide at $4^{\circ} \mathrm{C}$ for 
30 min. The labeled cell suspension was filtered and analyzed on Becton Dickinson FACSCalibur.

Cell proliferation was assayed using a Cell Counting Kit-8 (CCK-8, Dojindo Kumamoto Prefecture, Japan) according to the manufacturer's instructions. Briefly, cells were seeded in 96-well plates. At the indicated times, $10 \mu \mathrm{L}$ of the CCK- 8 solution was added into each well and then incubated for $2 \mathrm{~h}$ at $37^{\circ} \mathrm{C}$. The absorbance was measured by GENios Pro reader (Tecan, Switzerland) at the wavelength of $450 \mathrm{~nm}$.

\subsection{Nuclear staining}

Cells were fixed in $4 \%$ paraformaldehyde for $10 \mathrm{~min}$ and permeabilized with $0.2 \%$ Triton X-100 for $20 \mathrm{~min}$. Cells were incubated with solution containing DAPI for $5 \mathrm{~min}$ and then to visualize their nuclei by a laser scanning confocal microscope (Leica, Germany).

\subsection{Statistical analyses}

The data are expressed as mean \pm standard deviation (SD). Comparisons were analyzed by using Student's $t$-test or ANOVA. Differences were considered statistically significant at $P<0.05$. Analyses were performed using SPSS v.16.0 software.

\section{Results}

\subsection{Expression of geminin in VSMCs}

Because geminin is undetectable during G1 phase, and ac- cumulates during the $\mathrm{S}, \mathrm{G} 2$, and $\mathrm{M}$ phases in HeLa cell and MCF-7 $[8,16]$, we determined whether the pattern of the geminin expression was similar in rat VSMCs. As shown in Figure 1, the level of geminin expression was low in quiescent VSMCs (approximately $90 \%$ in G1), increased with the increase in cells transiting $\mathrm{S} / \mathrm{G} 2 / \mathrm{M}$ after serum restimulation, and then decreased as cells exited from $\mathrm{S} / \mathrm{G} 2 / \mathrm{M}$.

We next determined the expression levels of geminin in cells treated with angiotensin II and NE, which are major contributors to cardiovascular disease. As shown in Figure 2, angiotensin II $\left(10^{-2} \mu \mathrm{mol} \mathrm{L}{ }^{-1}\right)$ and $\mathrm{NE}\left(1 \mu \mathrm{mol} \mathrm{L}{ }^{-1}\right)$ significantly stimulated the proliferation of VSMCs and the expression of geminin.

\subsection{Efficiency of methods for silencing and over- expressing geminin in VSMCs}

To determine the role of geminin in the proliferation of VSMCs, we infected cells with adenoviruses expressing a cDNA encoding geminin and a geminin-specific shRNA. To establish the optimum conditions for inhibiting geminin expression, we cotransfected $293 \mathrm{~T}$ cells with the pEGFPN1-geminin expression vector and one of three distinct geminin-specific siRNAs. Geminin expression was inhibited most effectively by the siRNA designated 738 (Figure 3A). We next constructed an adenovirus vector expressing a geminin-specific shRNA using this siRNA sequence. Figure 3B shows that the expression of geminin in VSMCs infected with adenovirus vectors Ad-geminin and Ad-shRNA was significantly increased and decreased compared with controls Ad-null and Ad-non-silence at 24 and $48 \mathrm{~h}$ after serum restimulation, respectively.

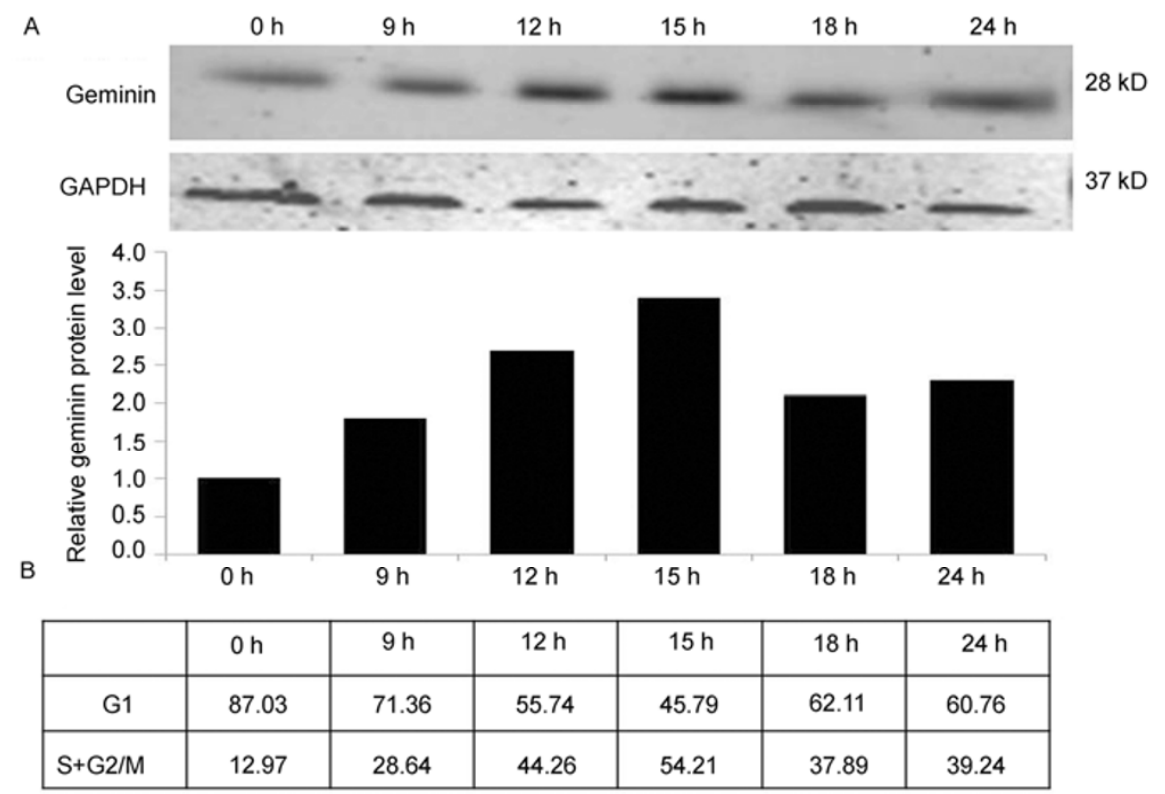

Figure 1 Analysis of geminin expression during the VSMC cell cycle. A, Western blot analysis of geminin expression in cells during G1 and S+G2/M. Cells were synchronized as described in Materials and methods and sampled at the indicated times. GAPDH served as a loading control. Geminin expression levels are normalized to the $0 \mathrm{~h}$ value. B, Analysis of the VSMC cell cycle. 

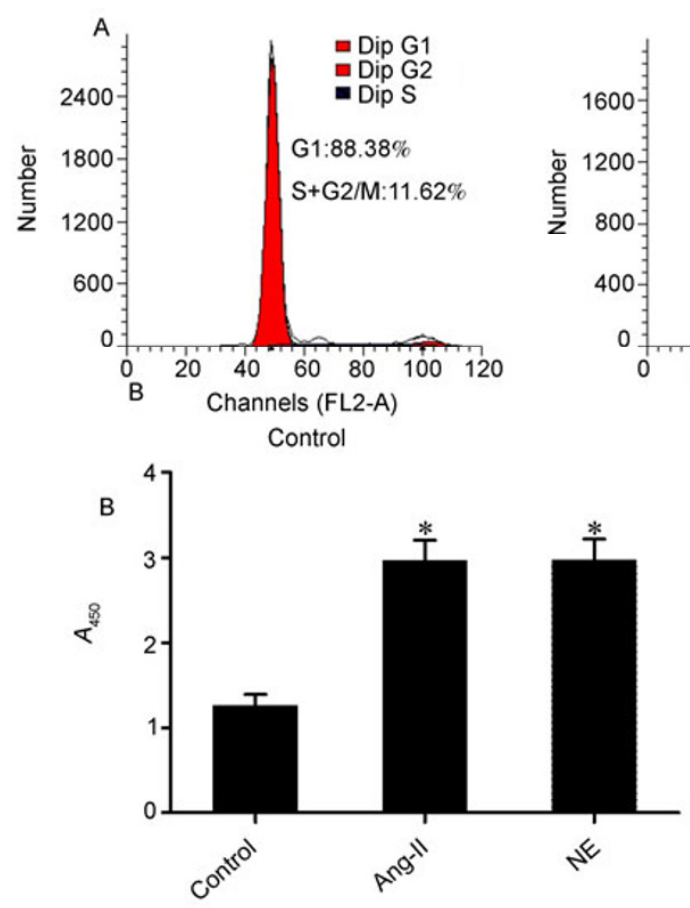
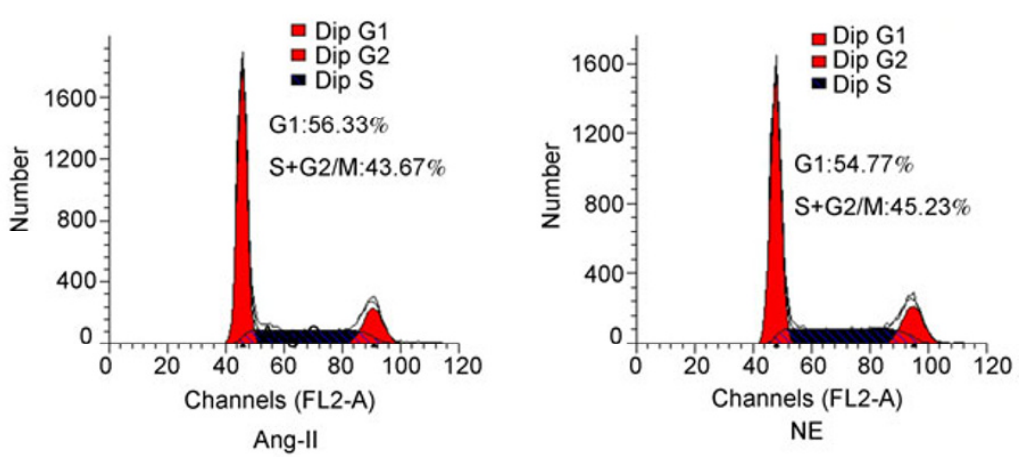

NE
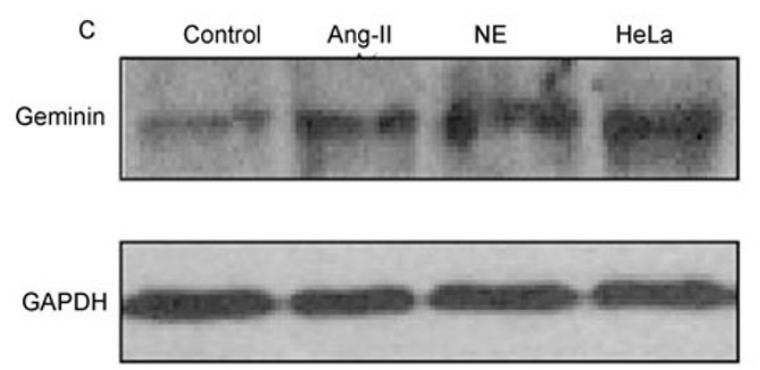

Figure 2 Analysis of geminin expression in cells treated with Ang-II and NE. Synchronous populations of quiescent VSMCs were treated with Ang-II and $\mathrm{NE}$ as described in Materials and methods. A, Cell cycle analysis. B, CCK-8 cell proliferation assay. ${ }^{*}, P<0.01$ (Ang-II or NE vs. control). C, Western blot analysis of geminin expression in untreated and Ang-II and NE treated cells. HeLa cells served as a positive control. The figure shows one of three independent experiments.

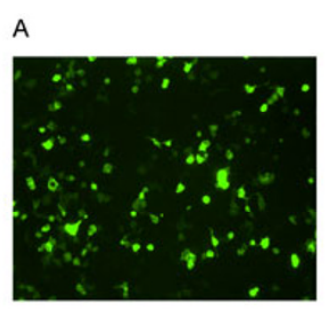

pEGFP-N1-geminin

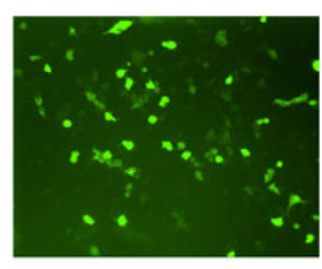

pEGFP-N1-geminin $+N C$ siRNA

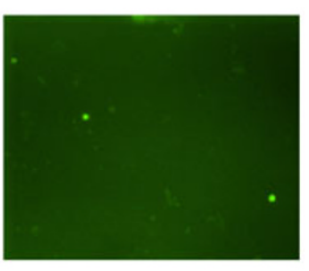

pEGFP-N1-geminin $+738$

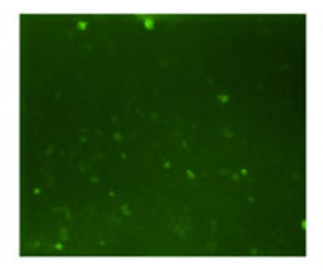

pEGFP-N1-geminin $+417$

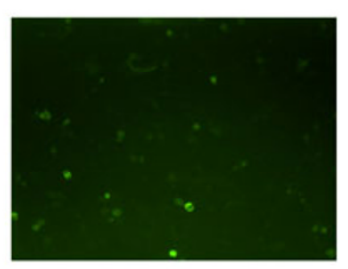

pEGFP-N1-geminin $+658$

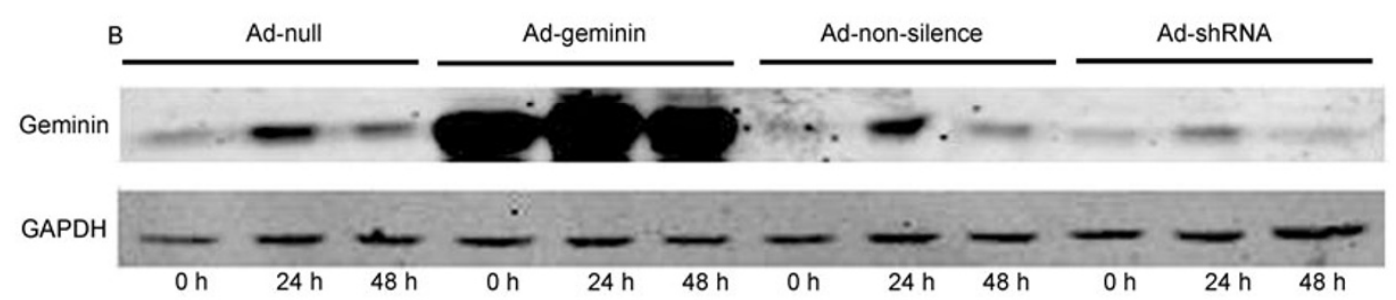

Figure 3 Evaluation of methods for silencing or overexpressing geminin in VSMCs. A, Effect of different geminin siRNAs on geminin expression. GFP fluorescence was detected as described in Materials and methods. B, Western blot analysis of geminin overexpression and inhibition by RNA silencing. VSMCs were infected with adenoviral expression vectors (Ad-null, Ad-geminin, Ad-non-silence, and Ad-shRNA) as described in Materials and methods. pEGFP-N1-geminin vector for expressing an EGFP-geminin fusion protein. NC, negative control.

\subsection{Effects of geminin on the proliferation of VSMCs}

The ability to inhibit or induce geminin expression in VSMCs using RNAi or transgene expression, respectively, facilitated our efforts to determine the effects of geminin on DNA replication, cell cycle progression, and proliferation. We found that either inhibition of geminin expression or overexpression did not significantly affect the DNA content or nuclear morphology of VSMCs compared with controls. Moreover, the percentage of cells at different phases of the cell cycle and the proliferative ability of VSMCs were also similar to the control values (Figures 4-6, Table 1). Because the expression levels of geminin fluctuate during the 

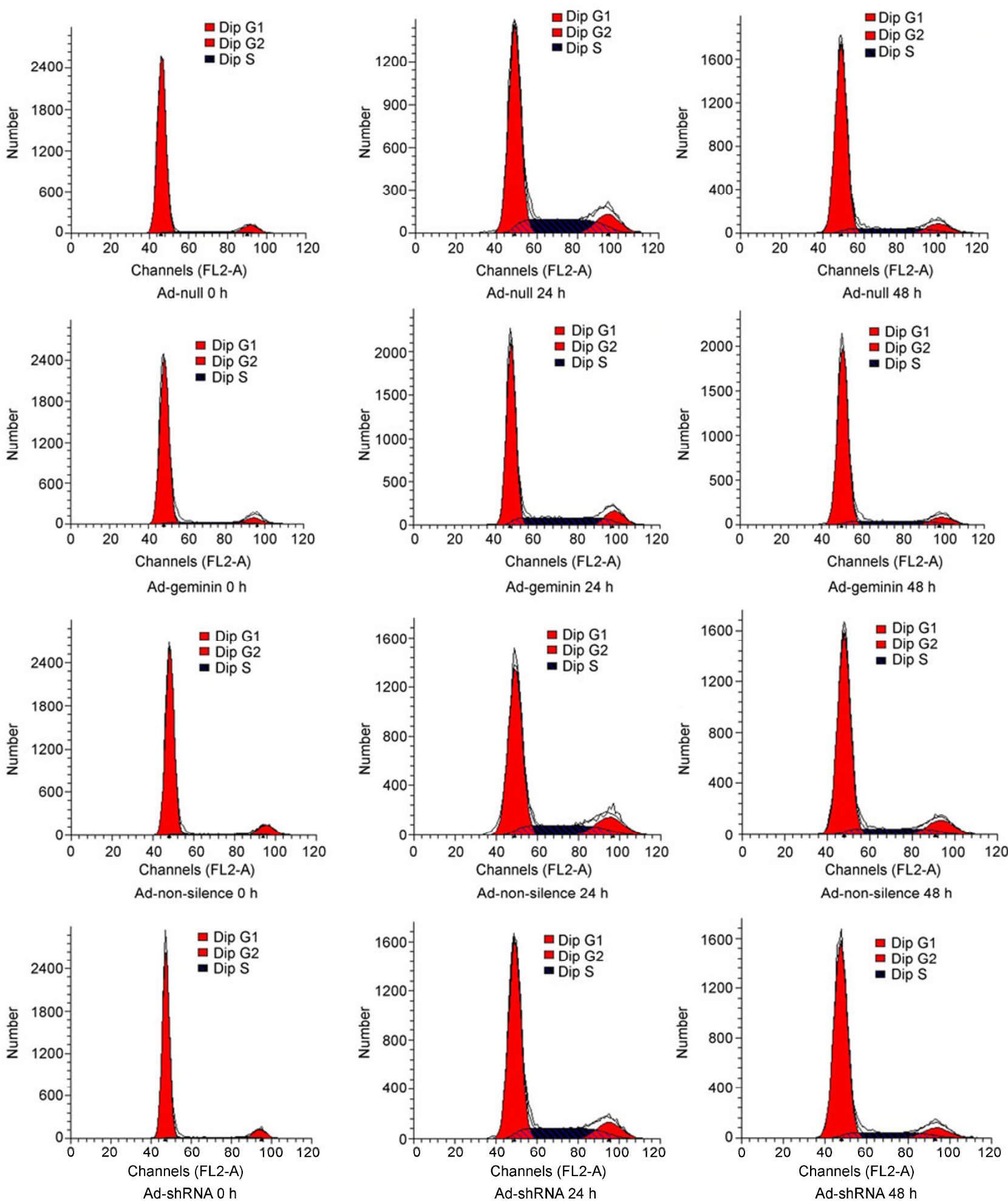

Figure 4 Analysis of DNA content using flow cytometry. VSMCs were infected with adenoviral vectors (Ad-null, Ad-geminin, Ad-non-silence, and Ad-shRNA) and stained with propidium iodide as described in Materials and methods. A minimum of 10000 events were analyzed.

cell cycle [8] and the peak is at $15 \mathrm{~h}$ in the present study, we also determined DNA content, nuclear morphology and proliferation at $15 \mathrm{~h}$ after inhibiting geminin expression and did not detect significant differences compared with the controls, the results were also similarly compared with control (Figures S1-S3, Table S1 in Supporting Information). 


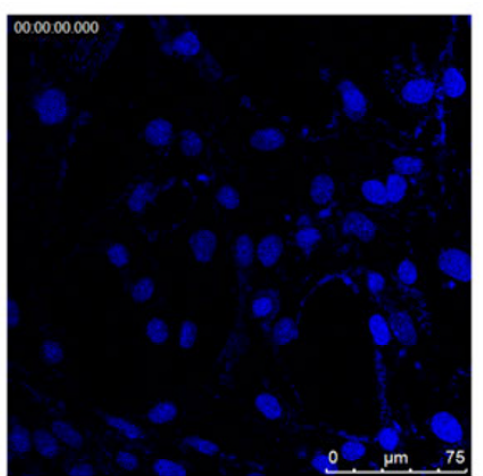

Ad-null $0 \mathrm{~h}$

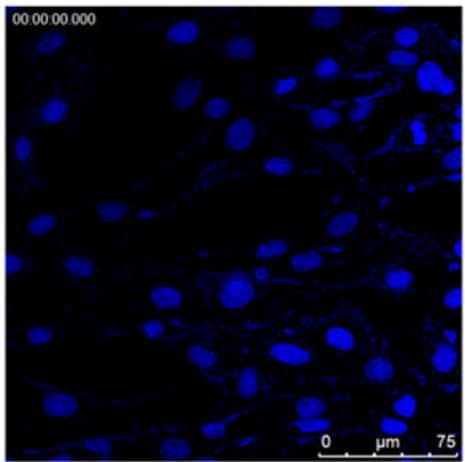

Ad-geminin $0 \mathrm{~h}$

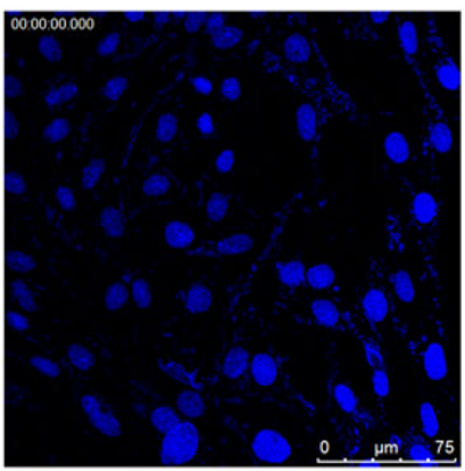

Ad-non-silence $\mathrm{Oh}$

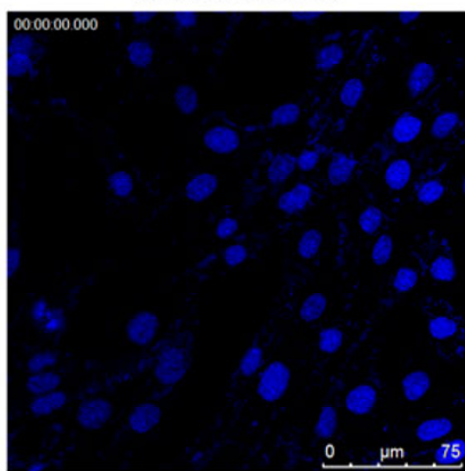

Ad-shRNA $0 \mathrm{~h}$

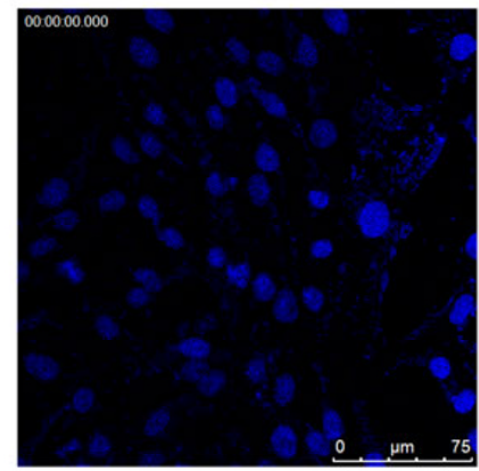

Ad-null $24 \mathrm{~h}$

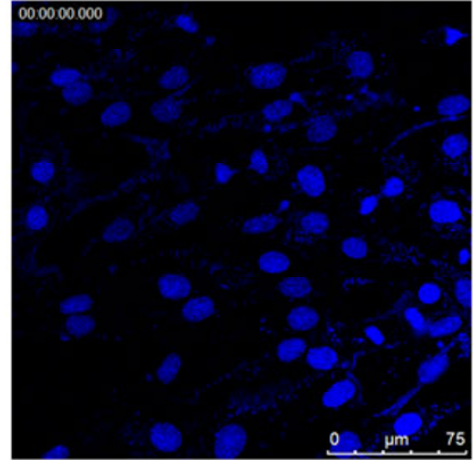

Ad-geminin $24 \mathrm{~h}$

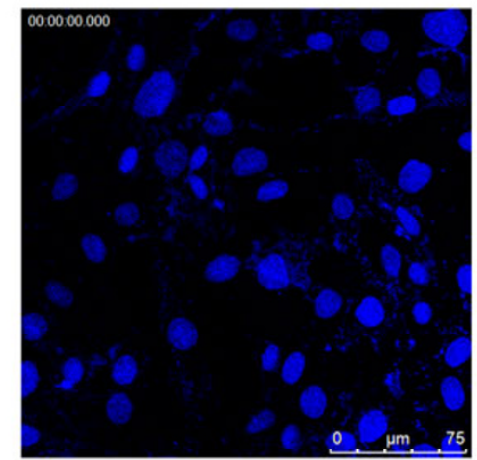

Ad-non-silence $24 \mathrm{~h}$

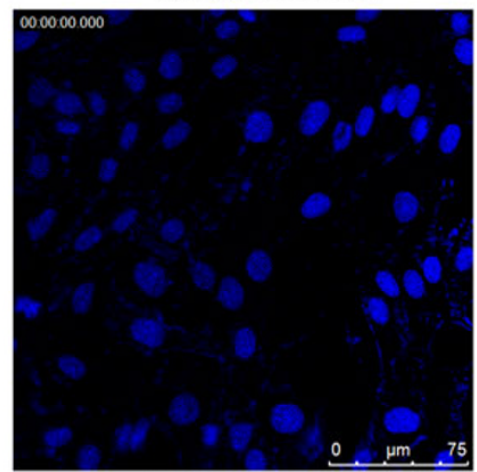

Ad-shRNA $24 \mathrm{~h}$

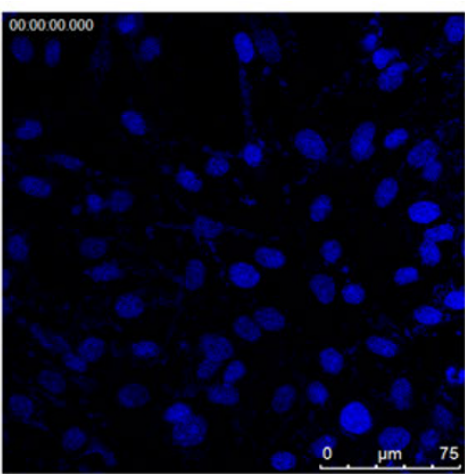

Ad-null $48 \mathrm{~h}$

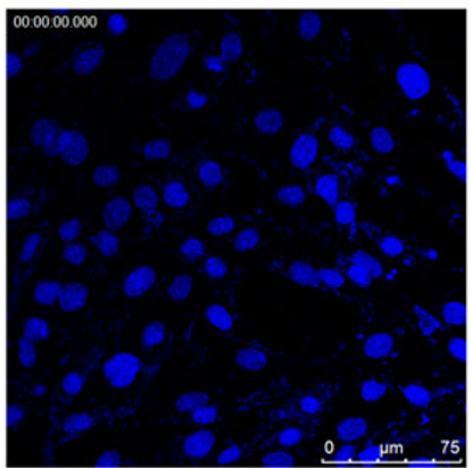

Ad-geminin $48 \mathrm{~h}$

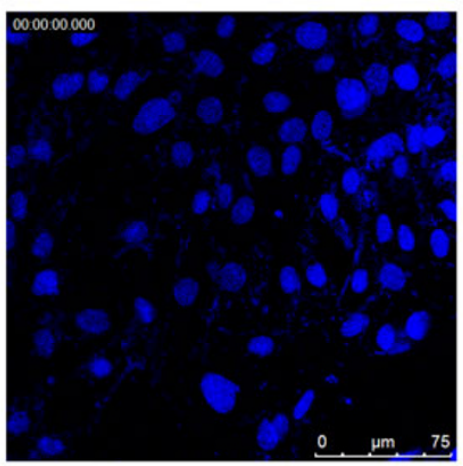

Ad-non-silence $48 \mathrm{~h}$

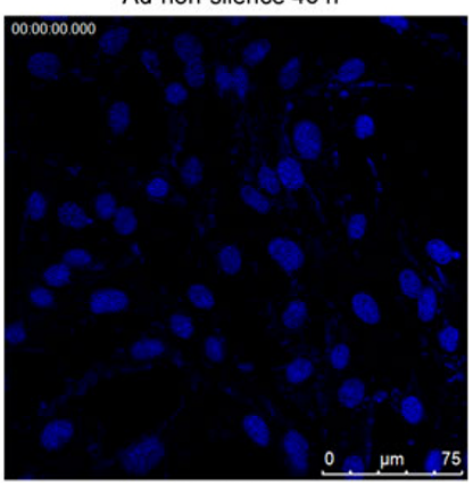

Ad-shRNA $48 \mathrm{~h}$

Figure 5 Nuclear morphology of VSMCs infected with Ad-null, Ad-geminin, Ad-non-silence, and Ad-shRNA at 0, 24, and 48 h following restimulation with serum. Cells were stained with 4',6-diamidino-2-phenylindole to visualize nuclei using a laser scanning confocal microscope. The figure shows one of three independent experiments. 
Table 1 Distribution of VSMCs in different stages of the cell cycle in Ad-null, Ad-geminin, Ad-non-silence, Ad-shRNA groups at 0, 24 and 48 h after serum restimulation

\begin{tabular}{|c|c|c|c|c|}
\hline Groups & & G1 (\%) & $\mathrm{S}(\%)$ & $\mathrm{G} 2 / \mathrm{M}(\%)$ \\
\hline Ad-null & \multirow{3}{*}{$0 \mathrm{~h}$} & $86.48 \pm 1.64$ & $5.70 \pm 1.01$ & $7.82 \pm 0.68$ \\
\hline Ad-geminin & & $87.41 \pm 2.80$ & $6.17 \pm 1.15$ & $6.43 \pm 1.72$ \\
\hline Ad-non-silence & & $87.51 \pm 1.52$ & $5.90 \pm 0.63$ & $6.59 \pm 2.08$ \\
\hline Ad-null & \multirow{3}{*}{$24 \mathrm{~h}$} & $65.14 \pm 1.54$ & $24.21 \pm 1.34$ & $10.64 \pm 0.69$ \\
\hline Ad-geminin & & $64.98 \pm 2.6$ & $23.99 \pm 1.39$ & $11.04 \pm 1.24$ \\
\hline Ad-non-silence & & $65.02 \pm 2.08$ & $23.28 \pm 3.2$ & $11.69 \pm 2.04$ \\
\hline Ad-null & \multirow{4}{*}{$48 \mathrm{~h}$} & $78.67 \pm 2.19$ & $12.73 \pm 1.01$ & $8.59 \pm 1.22$ \\
\hline Ad-geminin & & $78.98 \pm 2.21$ & $12.61 \pm 0.96$ & $8.40 \pm 1.34$ \\
\hline Ad-non-silence & & $79.26 \pm 2.45$ & $11.95 \pm 2.52$ & $8.79 \pm 0.43$ \\
\hline Ad-shRNA & & $77.84 \pm 1.38$ & $12.69 \pm 0.66$ & $9.46 \pm 1.63$ \\
\hline
\end{tabular}

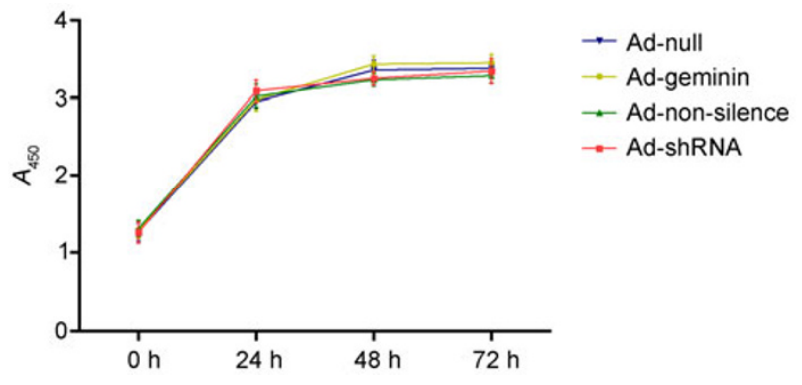

Figure 6 Effect of geminin on VSMC proliferation. Cells were infected with adenoviral vectors (Ad-null, Ad-geminin, Ad-non-silence, and Ad-shRNA) as described in Materials and methods. The absorbance of cells was measured at $450 \mathrm{~nm}$ at $0,24,48$, and $72 \mathrm{~h}$ after restimulation with serum.

\section{Discussion}

By interacting with Cdt1, geminin acts to prevent the relicensing of replication origins after they have fired once and accumulating evidence indicates that geminin plays an important role in maintaining the integrity of the genome. Proliferating normal cells, such as lymphocytes, male germ cells and epithelial cells, and cancer cell lines, express high levels of geminins. Depletion of geminin leads to additional rounds of replication in some cancer cells while overexpression of geminin stimulates progression through G1-S. In the present study, we show that VSMCs expressed geminin at low levels in G1 phase and accumulated it during S, G2, and $\mathrm{M}$ phases. Angiotensin II and NE, which are important factors in the pathogenesis of vascular proliferative diseases, also stimulated the VSMCs to express high levels of geminin, which supports its role in these diseases. However, inhibiting or overexpressing geminin in VSMCs did not significantly alter DNA content, nuclear morphology, the distribution of cells in the G1, S, G2/M phases or proliferative capacity compared with controls. Therefore, we conclude that geminin is dispensable for the regulation of VSMC proliferation.

We note that studies showing that DNA rereplication is induced by depleting geminin from eukaryotic cells were performed using the HCT116 human colorectal cancer cell line, the H1299 human lung cancer cell line, the US2OS human osteosarcoma cell line as well as others $[7,9,10]$. In contrast, the findings of our present studies using VSMCs are not consistent with the results of these studies. Therefore, the role of geminin in the regulation of the cell cycle machinery may be different in normal tissues or nontumorigenic cell lines from that in cancer cell lines. Although we did not further determine why the geminin was dispensable for regulation of DNA replication in VSMCs, we hypothesize the mechanism of that as follows. Cdt1 activity is the rate-limiting step in pre-RC assembly, and that geminin is the critical regulator of Cdt1 activity. Cdt1 activity is also regulated by other mechanisms in addition to geminin: (i) Cdt1 is phosphorylated by cyclin A-dependent kinases (cyclin A/Cdk1 and cyclin A/Cdk2), causing Cdt1 to bind the F-box protein Skp2, a component of the Skp1-Cullin1-Fbox protein ubiquitin ligase complex, Skp2 then degrades Cdt1. (ii) Cdt1 is regulated by Cul4-based ubiquitin ligaseand PCNA-mediated proteolysis during $S$ phase [17]. In normal cells, these mechanisms contribute to restricting genome duplication to once per cell division. Our findings that inhibiting geminin expression in VSMCs did not induce DNA rereplication in contrast to certain cancer cell lines suggest that the two pathways described above are inactive in cancer cell lines, but are active in VSMCs and compensate for the loss of geminin function.

Enforced overexpression of geminin stimulates cell proliferation and mediates an increase in the percentage of cells in $\mathrm{S}$ phase and is associated with a reduction in the percentage of the cells in G0/G1 phase [11]. In contrast, in the present study, the percentage of VSMCs at different stages of the cell cycle was not significantly altered compared with controls whether geminin was depleted or overexpressed.

Geminin is expressed at high levels in cancer cell lines of different histological origins as well as in primary colon, rectal, and breast tumors [11]. Primary breast cancers overexpress geminin at an early stage of progression suggesting that geminin plays an important role in initiating tumorigenesis [11]. Moreover, increased geminin expression is a 
powerful independent indicator of adverse prognosis in invasive breast cancer [16] and analysis of geminin expression may be useful for predicting the prognosis of patients with colorectal cancer [18]. These findings provide compelling evidence that geminin initiates and sustains malignant transformation, which is in stark contrast to our present results demonstrating that geminin was dispensable for the proliferation of VSMCs. Therefore, the difference between the role of geminin in the proliferation of cancer and normal cells suggests that it can be targeted to tumors without adverse effects on normal tissues.

In conclusion, geminin is expressed during the $\mathrm{S}, \mathrm{G} 2$, and $\mathrm{M}$ phases of the cell cycle in VSMCs but is dispensable for their proliferation in contrast to cell lines derived from human cancers. This apparent phenotype-specific difference in geminin function between normal and cancer cells implies that specific targeting of geminin in tumor cells may be a potential therapeutic strategy for treating cancers.

This work was supported by Beijing Municipal Natural Science Foundation (5102040).

1 Kawai-Kowase K, Owens G K. Multiple repressor pathways contribute to phenotypic switching of vascular smooth muscle cells. Am J Physiol Cell Physiol, 2007, 292: C59-69

2 Owens G K, Kumar M S, Wamhoff B R. Molecular regulation of vascular smooth muscle cell differentiation in development and disease. Physiol Rev, 2004, 84: 767-801

3 Charron T, Nili N, Strauss B H. The cell cycle: a critical therapeutic target to prevent vascular proliferative disease. Can J Cardiol, 2006, 22(Suppl B): 41B-55B

4 Stillman B. Cell cycle control of DNA replication. Science, 1996, 274: 1659-1664
5 Dutta A, Bell S P. Initiation of DNA replication in eukaryotic cells. Ann Rev Cell Dev Biol, 1997, 13: 293-332

6 Wohlschlegel J A, Dwyer B T, Dhar S K, et al. Inhibition of eukaryotic DNA replication by geminin binding to cdt1. Science, 2000, 290: 2309-2312

7 Mihaylov I S, Kondo T, Jones L, et al. Control of DNA replication and chromosome ploidy by geminin and cyclin A. Mol Cell Biol, 2002, 22: 1868-1880

8 McGarry T J, Kirschner M W. Geminin, an inhibitor of DNA replication, is degraded during mitosis. Cell, 1998, 93: 1043-1053

9 Zhu W, Chen Y, Dutta A. Rereplication by depletion of geminin is seen regardless of p53 status and activates a G2/M checkpoint. Mol Cell Biol, 2004, 24: 7140-7150

10 Melixetian M, Ballabeni A, Masiero L, et al. Loss of geminin induces rereplication in the presence of functional p53. J Cell Biol, 2004, 165: 473-482

11 Montanari M, Boninsegna A, Faraglia B, et al. Increased expression of geminin stimulates the growth of mammary epithelial cells and is a frequent event in human tumors. J Cell Physiol, 2005, 202: 215-222

12 Wohlschlegel J A, Kutok J L, Weng A P, et al. Expression of geminin as a marker of cell proliferation in normal tissues and malignancies. Am J Pathol, 2002, 161: 267-273

13 Eward K L, Obermann E C, Shreeram S, et al. DNA replication licensing in somatic and germ cells. J Cell Sci, 2004, 117: 5875-5886

14 Montanari M, Macaluso M, Cittadini A, et al. Role of geminin: from normal control of DNA replication to cancer formation and progression? Cell Death Diff, 2006, 13: 1052-1056

15 Pang X, Sun N L. Calcineurin-NFAT signaling is involved in phenylephrine-induced vascular smooth muscle cell proliferation. Acta Pharmacol Sin, 2009, 30: 537-544

16 Gonzalez M A, Tachibana K E, Chin S F, et al. Geminin predicts adverse clinical outcome in breast cancer by reflecting cell-cycle progression. J Pathol, 2004, 204: 121-130

17 Fujita M. Cdt1 revisited: complex and tight regulation during the cell cycle and consequences of deregulation in mammalian cells. Cell Div, 2006, 1: 22

18 Nishihara K, Shomori K, Tamura T, et al. Immunohistochemical expression of geminin in colorectal cancer: implication of prognostic significance. Oncol Rep, 2009, 21: 1189-1195

Open Access This article is distributed under the terms of the Creative Commons Attribution License which permits any use, distribution, and reproduction in any medium, provided the original author(s) and source are credited.

\section{Supporting Information}

Table S1 Effect of geminin in VSMCs proliferation

Figure S1 Western blot analysis for efficiency of geminin knocking down.VSMCs were infected with adenoviral vectors (Ad-non-silence, Ad-shRNA) in DMEM containing $0.5 \%$ FBS for $24 \mathrm{~h}$. The medium was then changed to DMEM containing $10 \%$ FBS and the cells were harvested at 0 and $15 \mathrm{~h}$ following serum restimulation.

Figure S2 DNA content was analyzed by flow cytometric analysis and cells distribution in different phases of the cell cycle. VSMCs were infected with adenoviral vectors (Ad-non-silence, Ad-shRNA) in DMEM containing 0.5\% FBS for $24 \mathrm{~h}$. The medium was then changed to DMEM containing 10\% FBS and harvested at 0 and $15 \mathrm{~h}$ following serum re-stimulation. The cells were trypsinized and washed, and DNA was stained with propidium iodide. Cell cycle analysis was performed with a minimum of 10000 events per analysis by using flow cytometric analysis.

Figure S3 The nuclei morphology of VSMCs infected with Ad-non-silence, Ad-shRNA at 0 and 15 h following serum restimulation. Cells were stained with DAPI to visualize their nuclei by laser scanning confocal microscope.

The supporting information is available online at life.scichina.com and www.springerlink.com. The supporting materials are published as submitted, without typesetting or editing. The responsibility for scientific accuracy and content remains entirely with the authors. 